To appear in Current Directions in Psychological Science

DOI: $10.1177 / 09637214211059536$

\title{
Ten Lessons about infants' everyday experiences
}

\author{
Kaya de Barbaro and Caitlin M. Fausey
}

Corresponding Authors:

Kaya de Barbaro, Department of Psychology, The University of Texas at Austin Email: kaya@austin.utexas.edu

Caitlin M. Fausey, Department of Psychology, University of Oregon Email: fausey@uoregon.edu 


\begin{abstract}
Audio recorders, accelerometers, and cameras that infants wear throughout their everyday lives capture the experiences that are available to shape development. Using sensors to capture behaviors in natural settings can reveal patterns within the everyday hubbub that are unknowable using methods that capture shorter, more isolated, or more planned slices of behavior. Here, we review 10 lessons learned from recent endeavors in which researchers neither designed nor participated in infants' experiences and instead quantified patterns that arose within infants' own spontaneously arising everyday experiences. The striking heterogeneity of experiences-the fact that there is no meaningfully "representative" hour of a day, instance of a category, interaction context, or infant-inspires next steps in theory and practice that embrace the complex, dynamic, and multiple pathways of human development.
\end{abstract}

Keywords: infancy, wearable sensors, ecology, everyday activities, development 


\section{Ten Lessons about infants' everyday experiences}

Imagine a day in the life of an infant who is uniformly well fed and alert and stays in the same sparsely furnished room from morning to evening, playing with the same object while hearing the same words from their caregiver on loop. A striking contrast to this imagined monotony-the ups and downs of everyday interactions, complete with diaper changes, dishwashing, siblings screaming, bath times, and bedtimes-emerges from records of infants' activities captured by wearable sensors that accompany infants across space and time. Here, we review 10 lessons about infants' everyday experiences based on recent research that has quantified patterns of infants' sensory histories as they do, learn, and grow in their real lives.

Wearable sensors, such as audio recorders, accelerometers, and cameras that infants wear throughout their everyday lives, capture the experiences that are available to shape their development. These experiences are not scripted by developmental theorists nor perturbed by the presence of an outsider recording infants' behaviors. Wearable sensors thus offer the unique opportunity to capture the experiences on which a lot of learning is hypothesized to depend. Insights about these experiences raise new hypotheses and sharpen collective focus toward theories of developmental change centered on everyday heterogeneity.

The following lessons are things researchers have learned about the distribution of infants' experiences across space, time, and the complex structure of everyday settings. Our discussion of these lessons includes implications for learning and development when supported by available evidence. We then highlight connections to extant theories and point to new directions prompted by these lessons.

\section{The 10 Lessons}

\section{Infants do not repeat the same experience over and over}

Everyday experiences in infancy include multiple activities (Roy et al., 2015; Soderstrom \& Wittebolle, 2013), postures (Franchak, 2019), objects (Clerkin et al., 2017), speakers (Bergelson, Amatuni et al., 2019), and musical tunes (Mendoza \& Fausey, 2021). The full diversity of experiences cannot simultaneously occur every second of every day. Instead, experiences vary over time. For example, some hours and days are filled with more verbal chatter than others (Casillas et al., 2020; d'Apice et al., 2019). Extrapolating evidence from one timescale to another is also not straightforward. For example, a recent study about the number of distinct nouns (e.g., "cup," "bowl," "spoon," "cat," "dog") spoken near infants showed that, on average, infants heard roughly 75 different nouns during 1 continuous hour and roughly 200 different nouns across a full 10-hr recording day (Bergelson, Amatuni, et al., 2019). That is, infants encountered neither the same 75 nouns within every hour of the day nor 75 new nouns each hour of the day. Sensors capturing these dynamics are reinvigorating research about how developmental change arises from multiple timescales of cumulating experience.

\section{Infants encounter a lot of different things, but they encounter only a small set of things pervasively \\ Digging deeper into patterns of repetition and change during infants' experiences reveals a strikingly consistent pattern: Infants encounter some individual identities very much more often than others. For example, infants see many different people's faces, but they see a small number of these individuals' faces repeatedly (Jayaraman et al., 2015). Similarly, infants encounter a huge variety of objects during mealtime activities, but a small set of them, such as}


cups and spoons, are especially prevalent (Clerkin et al., 2017). Among the many distinct tunes that infants encounter, some tunes dominate a day's musical seconds whereas others are brief (Mendoza \& Fausey, 2021). The nonuniform nature of everyday distributions is inspiring new hypotheses about how people remember and generalize as they learn (Smith et al., 2018).

\section{Inputs are nested within everyday activities}

The objects, words, and interactions from which infants learn arise within everyday activities. For example, an infant sitting in a high chair during breakfast might see a cup and hear their father name it. Or the infant could encounter a cup while playing with siblings in their toy kitchen or notice a cup in a favorite picture book at bedtime. New insights about the power of activities for organizing learning have emerged from several recent investigations. For example, researchers now know that hearing a word in a consistent location and at a consistent time of day, over days and weeks, helps infants learn to say the word themselves (Roy et al., 2015). Another study has shown that holding objects generates first-person views of these objects that are variable and linked in time, which supports recognizing objects in new contexts (Bambach et al., 2018). Finally, caregivers' emotional availability during bedtime routines predicts infants' sleep quality (Teti et al., 2010). The context-specificity of this bedtime-sleep association is consistent with a role for activities in organizing opportunities for developmental change.

\section{Experiences change over developmental time}

Just as infants' interests and abilities change over time, so too do their everyday experiences. For example, 2-month-olds encounter faces during many more minutes per day than do 15month-olds (Jayaraman et al., 2015). And the faces in view for younger infants are more likely to be close to them and persistent in time than the faces in view for older infants (Jayaraman et al., 2015; Jayaraman \& Smith, 2019). In contrast, infants' views increasingly include hands, and hands holding objects, over developmental time (Fausey et al., 2016). Postures and motor activities also change over developmental time; rates of sitting increase and rates of being held decrease over the first year of infancy (Franchak, 2019). Limb dynamics, such as arm movements, become increasingly dependent on recent prior motor behaviors over the course of the first year of life (Abney et al., 2014). The extent to which other sensory histories, such as histories of access to everyday objects, language, and music, change over developmental time is an open question worthy of future investigation.

\section{Downtime is part of the everyday rhythm}

The reality that activities start and stop is readily observed in everyday life. Free-flowing behavioral streams consist of numerous activities of varying duration and switches befitting the attentional and emotional needs of infants and caregivers alike. Sometimes everyone is playing on the living room floor with objects, and other times caregivers wash dishes while silently monitoring their infants. Any single activity, including speech, therefore rises and falls over time. In fact, recent reports have revealed that roughly 20 to $30 \%$ of sampled everyday activity does not include any speech at all, in dramatic contrast to traditional laboratory samples, during which caregivers talk constantly (Cristia et al., 2021; Tamis-LeMonda et al., 2017). The contrast between behaviors in everyday life, and behaviors during a play session in a special-purpose room for a predetermined amount of time, highlights an emerging priority to account for the prevalence and rhythm of everyday activities in accounts of experience-dependent learning. 


\section{Some everyday rhythms take hours-not minutes-to arise}

Some patterns of activity take hours to unfold. For example, some caregivers might talk a little bit throughout an entire day, and this would never be knowable by sampling only 5 min.

Similarly, an infant crying for 5 min might affect a caregiver's mental health differently than an infant crying for $8 \mathrm{hr}$. Wearable sensors have recently captured such extended behaviors and their effects. For example, infants whose caregivers were consistently chatty, participating in at least one back-and-forth conversational turn with their infant every 5 min throughout the day, had lower symptoms of psychopathology as toddlers, compared with infants whose caregivers were not as consistently chatty. By contrast, the overall volume of caregiver speech did not predict symptoms of psychopathology in the toddlers (King et al., 2021). A study that quantified within-person changes in mothers' mood using ecological momentary assessments synchronized with infants' crying detected from everyday audio recordings showed that aboveaverage crying duration in the $10 \mathrm{~min}$ and $8 \mathrm{hr}$ prior to maternal mood report predicted worse mood among the mothers. However, only when infant crying was above average for $8 \mathrm{hr}$ did mothers show subsequent feelings of depression and lower self-efficacy relative to occasions in which infants cried less than average. Thus, in ecologically valid settings, the effects of infant crying on caregivers' mental health became apparent only at this longer, daylong timescale (de Barbaro et al., 2021).

\section{Everyday activities showcase heterogeneity in caregiving}

Expansive time, space, and caregiver responsibilities at home have implications for caregiving and its consequences. For example, individual differences in how caregivers structure physical space, and in how they encourage or restrain their infants' movements, can affect infants' opportunities to sample their environment (Rachwani et al., 2020). Other forms of everyday encouraging, such as praising infants for helping, vary across families as well (Dahl, 2015). Further, in addition to confirming well-established variation in the quality of caregiver-infant interactions, wearable sensors are revealing that caregivers vary in the total amount of time they spend directly engaging with their infants. For example, in one study, roughly half of families read to their infants at least once in a day, and about half did not (Clemens \& Kegel, 2021). Caregivers also differ in many dimensions of their availability to infants, such as in their physical presence, proximity, and physical contact (Rachwani et al., 2020; Yao et al., 2019). For example, in one study, the amount of time caregivers held their infants in a 45-min session ranged from 10 to 30 min (Yao et al., 2019). Finally, everyday holding matters for infants' distress, feeding, and sleep (Little et al., 2018; St James-Roberts et al., 2006).

\section{Experiences are not interchangeable across infants}

There is tremendous diversity within and across the world's communities in child-rearing beliefs, caregiving configurations, access to material resources, and many other circumstances that structure everyday life. Individual infants' experiences are therefore nested within many organizing levels of local context. Infant-to-infant variation in everyday language experiences has been extensively documented; individual infants of similar age are exposed to different daily rates of child-directed speech (Casillas et al., 2020; Weisleder \& Fernald, 2013), conversational back-and-forth with caregivers (Romeo et al., 2018), and dynamics of language over multiple timescales (d'Apice et al., 2019; King et al., 2021). Demographic realities such as maternal education and socioeconomic status have been associated with some of these variations. For example, on average, in North American families, mothers with higher levels of education are more talkative with their infants (Bergelson, Casillas, et al., 2019). Variation in the motor domain is also evident. For example, some infants walk up their caregivers' chests, whereas others lie bound for hours in cradles (Rachwani et al., 2020). The causes and consequences of infant-to- 
infant variation in everyday experiences are complex, as we highlight in the final two lessons.

\section{What you do is what you learn}

Experience-dependent learning pervades human development. For example, infants learn to speak the languages and recognize the faces in their everyday milieu. Recent discoveries have revealed links between detailed quantifications of everyday experiences and learning. One striking example is that infants who encounter more child-directed speech in their everyday lives process speech faster and have larger vocabularies (Weisleder \& Fernald, 2013), which has potential implications for school readiness. Connecting heard words to seen objects also matters, such that infants who more often see objects while they are being named are more likely to learn object names (Bergelson \& Aslin, 2017). Infants' earliest learned object names also tend to be the names of objects that they see pervasively (Clerkin et al., 2017). Finally, everyday opportunities to move independently accelerate sitting and walking (Rachwani et al., 2020). Overall, seeing, hearing, and doing drive development.

\section{There are multiple pathways to learning}

Links between quantified features of everyday experiences and learning do not always generalize straightforwardly across infants. For example, infants in Tseltal Mayan communities are exposed to lower rates of child-directed speech than are infants in upper-class Englishspeaking U.S. communities yet show similar developmental timing of early language skills (Casillas et al., 2020). The experiences that give rise to learning are necessarily specific to individual learners; no single infant encounters an "average" history of everyday experiences. If the varied features of everyday life cohere in different ways for different infants, then any single feature's impact on learning may also vary across infants. Researchers have a lot yet to discover, given that developmental pathways arise from multiple causes and multiple interacting processes (Samuelson, 2021).

\section{Building Next-Generation Theories of Developmental Change}

Fully understanding experience-dependent developmental change is a work in progress. We envision future research that construes everyday experiences as both the causes and consequences of developmental change over time, and in which lessons from multiple approaches are integrated to reveal mechanisms by which infants build skills over time.

Because infants' everyday skills may or may not overlap with the behavioral repertoires elicited in researcher-designed tasks or with caregivers' reports, any account of developmental change will benefit from continued investigations of the nature of everyday behaviors. For example, although stepping behavior as measured by walking on an elongated pressure mat provides an efficient and reliable assessment of motor development, it misses infants' omnidirectional steps observed when infants can roam around a room (Lee et al., 2018). Similarly, the ability to distinguish word referents in a two-alternative forced-choice task provides insight into early stages of word comprehension but obscures potential challenges and scaffolds for learning and identifying referents in everyday language use (Gogate et al., 2000). Therefore, characterizing everyday experience showcases skills that become the to-be-explained developmental achievements in theories. Additionally, reports of everyday behaviors can drive new hypotheses about relevant mechanisms for learning in context. Indeed, many of the operative mechanisms may be the everyday behaviors themselves as infants practice over time (Lockman \& TamisLeMonda, 2021). For example, infants' self-sitting experience shapes their visual perception 
skills (Soska et al., 2010). Although such everyday experiences are commonly presumed in theories of development, they are typically not directly assessed (Nastase et al., 2020). Thus, an important step en route to understanding how experiences matter for emerging skills is to characterize behaviors in context (Adolph, 2019).

Advancing understanding of precisely how everyday behaviors arise and matter for developmental change can also benefit from iterative synergies with researcher-constrained studies. For example, now that everyday distributions are known to be highly nonuniform in many domains, next-step researcher-constrained studies can investigate the impact of these everyday parameters on learning in traditional lab paradigms. Other synergistic innovations could merge everyday complexity with researcher-designed control. For example, manipulating free-flowing parent-child interactions can test the importance of parents' real-time multimodal feedback for language production (Goldstein \& Schwade, 2008). Similarly, manipulating infants' grasping experiences over weeks can be used to assess the importance of extended timescales of everyday experiences for learning (Needham et al., 2002).

Throughout these next steps, understanding about infants' everyday experiences will benefit from evidence across the world's diverse contexts. Much of the current evidence is drawn from White, Western-educated populations and cannot be considered evidence for universal lessons about the nature of infants' everyday experiences.

\section{Conclusion}

The use of sensors to capture everyday activity has revealed the complex and dynamic nature of the experiences available to shape infants' development. The striking heterogeneity of experiences across space, time, and contexts suggests three important priorities en route to creating a world that supports everyone's endeavors to learn and grow. First, the dynamic rising and falling of infants' opportunities to learn about the world should prompt researchers to sample ever-more-extended timescales in order to detect the frequency, variability, and organization of everyday experiences. Second, caregivers, clinicians, and policy makers may find inspiration and information in a base of evidence that increasingly approximates the realities of everyday life. Studies that compare behaviors from laboratory and everyday samples sometimes reveal generalizable insights and other times highlight certain realities that arise only in everyday life and may prompt updated practices (Tamis-LeMonda et al., 2017). Third, everyday experiences vary within and across communities, and this variation has implications for children's skills and opportunities over time. It can sometimes serve as a barometer for potential structural inequities that may shape everyday interactions and affect some communities more severely than others. Everyone dedicated to supporting healthy development would be wise to attend to these findings, pursue understanding of their multiple causes and consequences, and integrate ideas across areas of expertise in order to best serve all children. 


\section{Recommended Reading}

Adolph, K. E. (2019). (See References). Highlights interindividual and intraindividual variability, and multiple timescales, in processes of behavioral change.

de Barbaro, K. (2019). Automated sensing of daily activity: A new lens into development. Developmental Psychobiology, 61(3), 444-464. https://doi.org/10.1002/dev.21831. Reviews recent innovations in sensing as used to inform developmental theory.

Rogoff, B., Dahl, A., \& Callanan, M. (2018). The importance of understanding children's lived experience. Developmental Review, 50(Pt. A), 5-15. https://doi.org/10.1016/j.dr.2018.05.006. Presents a sociocultural perspective on children's participation in everyday practices of their own lives.

Rowe, M. L., \& Weisleder, A. (2020). Language development in context. Annual Review of Developmental Psychology, 2, 201-223. https://doi.org/10.1146/annurev-devpsych-042220-121816. Describes context as a set of nested systems and discusses policy implications of social-demographic variation in children's everyday environments.

Thelen, E., \& Smith, L.B. (1994). A dynamic systems approach to the development of cognition and action. MIT Press. Discusses general principles of nonlinear dynamic systems, including a new meaning for time, a new meaning for variability, and a new respect for individuality.

\section{Funding}

This work was supported by a National Institute of Mental Health K01 Award (1K01MH111957-01A1), awarded to K. de Barbaro. 


\section{References}

Abney, D. H., Warlaumont, A. S., Haussman, A., Ross, J. M., \& Wallot, S. (2014). Using nonlinear methods to quantify changes in infant limb movements and vocalizations. Frontiers in Psychology, 5, Article 771. https://doi.org/10.3389/fpsyg.2014.00771

Adolph, K. E. (2019). An ecological approach to learning in (not and) development. Human Development, 63(3-4), 180-201. https://doi.org/10.1159/000503823

Bambach, S., Crandall, D. J., Smith, L. B., \& Yu, C. (2018). Toddler-inspired visual object learning. In S. Bengio, H. Wallach, H. Larochelle, K. Grauman, N. Cesa-Bianchi, \& R. Garnett (Eds.), Advances in neural information processing systems 31 (NeurIPS 2018). Retrieved from https://proceedings.neurips.cc/paper/2018/file/48ab2f9b45957ab574cf005eb8a76760-Paper.pdf

Bergelson, E., Amatuni, A., Dailey, S., Koorathota, S., \& Tor, S. (2019). Day by day, hour by hour: Naturalistic language input to infants. Developmental Science, 22(1), Article e12715.

https://doi.org/10.1111/desc.12715

Bergelson, E., \& Aslin, R. N. (2017). Nature and origins of the lexicon in 6-mo-olds. Proceedings of the National Academy of Sciences, USA, 114(49), 12916-12921. https://doi.org/10.1073/pnas.1712966114

Bergelson, E., Casillas, M., Soderstrom, M., Seidl, A., Warlaumont, A. S., \& Amatuni, A. (2019). What do North American babies hear? A large-scale cross-corpus analysis. Developmental Science, 22(1), Article e12724. https://doi.org/10.1111/desc. 12724

Casillas, M., Brown, P., \& Levinson, S. C. (2020). Early language experience in a Tseltal Mayan village. Child Development, 91(5), 1819-1835. https://doi.org/10.1111/cdev.13349

Clemens, L. F., \& Kegel, C. A. (2021). Unique contribution of shared book reading on adult-child language interaction. Journal of Child Language, 48(2), 373-386.

https://doi.org/10.1017/S0305000920000331

Clerkin, E. M., Hart, E., Rehg, J. M., Yu, C., \& Smith, L. B. (2017). Real-world visual statistics and infants' first-learned object names. Philosophical Transactions of the Royal Society B: Biological Sciences, 372(1711), Article 20160055. http://doi.org/10.1098/rstb.2016.0055

Cristia, A., Lavechin, M., Scaff, C., Soderstrom, M., Rowland, C., Räsänen, O., Bunce, J., \& Bergelson, E. (2021). A thorough evaluation of the Language Environment Analysis (LENA) system. Behavior Research Methods, 53(2), 467-486. https://doi.org/10.3758/s13428-020-01393-5

Dahl, A. (2015). The developing social context of infant helping in two U.S. samples. Child Development, 86(4), 1080-1093. https://doi.org/10.1111/cdev.12361

d'Apice, K., Latham, R. M., \& von Stumm, S. (2019). A naturalistic home observational approach to children's language, cognition, and behavior. Developmental Psychology, 55(7), 1414-1427.

https://doi.org/10.1037/dev0000733

de Barbaro, K., Micheletti, M., Yao, X., Khante, P., Johnson, M., \& Goodman, S. (2021). Sensing a day in Mom's Life: Objective markers of everyday experiences predict real-time fluctuations in maternal mental health [Manuscript submitted for publication]. Department of Psychology, University of Texas at Austin.

Fausey, C. M., Jayaraman, S., \& Smith, L. B. (2016). From faces to hands: Changing visual input in the first two years. Cognition, 152, 101-107. https://doi.org/10.1016/j.cognition.2016.03.005

Franchak, J. M. (2019). Changing opportunities for learning in everyday life: Infant body position over the first year. Infancy, 24(2), 187-209. https://doi.org/10.1111/infa.12272 
Gogate, L. J., Bahrick, L. E., \& Watson, J. D. (2000). A study of multimodal motherese: The role of temporal synchrony between verbal labels and gestures. Child Development, 71(4), 878-894. https://doi.org/10.1111/1467-8624.00197

Goldstein, M. H., \& Schwade, J. A. (2008). Social feedback to infants' babbling facilitates rapid phonological learning. Psychological Science, 19(5), 515-523. https://doi.org/10.1111/j.14679280.2008.02117.x

Jayaraman, S., Fausey, C. M., \& Smith, L. B. (2015). The faces in infant-perspective scenes change over the first year of life. PLOS ONE, 10(5), Article e0123780. https://doi.org/10.1371/journal.pone.0123780

Jayaraman, S., \& Smith, L. B. (2019). Faces in early visual environments are persistent not just frequent. Vision Research, 157, 213-221. https://doi.org/10.1016/j.visres.2018.05.005

King, L. S., Querdasi, F. R., Humphreys, K. L., \& Gotlib, I. H. (2021). Dimensions of the language environment in infancy and symptoms of psychopathology in toddlerhood. Developmental Science, 24(5), Article e13082. https://doi.org/10.1111/desc.13082

Lee, D. K., Cole, W. G., Golenia, L., \& Adolph, K. E. (2018). The cost of simplifying complex developmental phenomena: A new perspective on learning to walk. Developmental Science, 21(4), Article e12615. https://doi.org/10.1111/desc.12615

Little, E. E., Legare, C. H., \& Carver, L. J. (2018). Mother-infant physical contact predicts responsive feeding among U.S. breastfeeding mothers. Nutrients, 10(9), Article 1251.

https://doi.org/10.3390/nu10091251

Lockman, J. J., \& Tamis-LeMonda, C. S. (2021). Young children's interactions with objects: Play as practice and practice as play. Annual Review of Developmental Psychology, 3. Advance online publication. https://doi.org/10.1146/annurev-devpsych-050720-102538

Mendoza, J. K., \& Fausey, C. M. (2021). Everyday music in infancy. Developmental Science, 24(6), Article e13122. https://doi.org/10.1111/desc.13122

Nastase, S. A., Goldstein, A., \& Hasson, U. (2020). Keep it real: Rethinking the primacy of experimental control in cognitive neuroscience. Neurolmage, 222, Article 117254.

https://doi.org/10.1016/j.neuroimage.2020.117254

Needham, A., Barrett, T., \& Peterman, K. (2002). A pick-me-up for infants' exploratory skills: Early simulated experiences reaching for objects using 'sticky mittens' enhances young infants' object exploration skills. Infant Behavior and Development, 25(3), 279-295. https://doi.org/10.1016/S01636383(02)00097-8

Rachwani, J., Hoch, J. E., \& Adolph, K. E. (2020). Action in development: Variability, flexibility, and plasticity. In J. J. Lockman \& C. S. Tamis-LeMonda (Eds.), The Cambridge handbook of infant development: Brain, behavior, and cultural context (pp. 469-494). Cambridge University Press.

Romeo, R. R., Leonard, J. A., Robinson, S. T., West, M. R., Mackey, A. P., Rowe, M. L., \& Gabrieli, J. D. E. (2018). Beyond the 30-million-word gap: Children's conversational exposure is associated with language-related brain function. Psychological Science, 29(5), 700-710.

https://doi.org/10.1177/0956797617742725

Roy, B. C., Frank, M. C., DeCamp, P., Miller, M., \& Roy, D. (2015). Predicting the birth of a spoken word. Proceedings of the National Academy of Sciences, USA, 112(41), 12663-12668.

http://doi.org/10.1073/pnas.1419773112 
Samuelson, L. K. (2021). Toward a precision science of word learning: Understanding individual vocabulary pathways. Child Development Perspectives, 15(2), 117-124.

https://doi.org/10.1111/cdep.12408

Smith, L. B., Jayaraman, S., Clerkin, E., \& Yu, C. (2018). The developing infant creates a curriculum for statistical learning. Trends in Cognitive Sciences, 22(4), 325-336.

https://doi.org/10.1016/j.tics.2018.02.004

Soderstrom, M., \& Wittebolle, K. (2013). When do caregivers talk? The influences of activity and time of day on caregiver speech and child vocalizations in two childcare environments. PLOS ONE, 8(11), Article e80646. https://doi.org/10.1371/journal.pone.0080646

Soska, K. C., Adolph, K. E., \& Johnson, S. P. (2010). Systems in development: Motor skill acquisition facilitates three-dimensional object completion. Developmental Psychology, 46(1), 129-138.

https://doi.org/10.1037/a0014618

St James-Roberts, I., Alvarez, M., Csipke, E., Abramsky, T., Goodwin, J., \& Sorgenfrei, E. (2006). Infant crying and sleeping in London, Copenhagen and when parents adopt a "proximal" form of care.

Pediatrics, 117(6), e1146-e1155. https://doi.org/10.1542/peds.2005-2387

Tamis-LeMonda, C. S., Kuchirko, Y., Luo, R., Escobar, K., \& Bornstein, M. H. (2017). Power in methods: Language to infants in structured and naturalistic contexts. Developmental Science, 20(6), Article e12456. https://doi.org/10.1111/desc.12456

Teti, D. M., Kim, B.-R., Mayer, G., \& Countermine, M. (2010). Maternal emotional availability at bedtime predicts infant sleep quality. Journal of Family Psychology, 24(3), 307-315.

https://doi.org/10.1037/a0019306

Weisleder, A., \& Fernald, A. (2013). Talking to children matters: Early language experience strengthens processing and builds vocabulary. Psychological Science, 24(11), 2143-2152.

https://doi.org/10.1177/0956797613488145

Yao, X., Plötz, T., Johnson, M., \& de Barbaro, K. (2019). Automated detection of infant holding using wearable sensing: Implications for developmental science and intervention. Proceedings of the ACM on Interactive, Mobile, Wearable and Ubiquitous Technologies, 3(2), Article 64.

https://doi.org/10.1145/3328935 